\title{
NONVANISHING OF THE DIFFERENTIAL OF HOLOMORPHIC MAPPINGS AT BOUNDARY POINTS
}

\author{
M. S. Baouendi, Xiaojun Huang, and Linda Preiss Rothschild
}

\section{$\S 0$ Introduction}

Let $M$ and $M^{\prime}$ be two smooth hypersurfaces in $\mathbb{C}^{n}$. A smooth mapping $h: M \rightarrow M^{\prime}$ is a $C R$ mapping if its components are annihilated by the induced Cauchy-Riemann operator on $M$. Let $p_{0} \in M$ and suppose that near $p_{0}, h$ is the restriction of a holomorphic mapping $H$ defined on one side of $M$ near $p_{0}$ and smooth up to $M$. We shall say that $h$ satisfies the Hopf lemma property at $p_{0}$ if the component of $H$ normal to $M_{2}$ has a nonzero derviative at $p_{0}$ in the normal direction to $M_{1}$. The hypersurface $M$ is said to be minimal at $p_{0} \in M$ if there is no germ of a complex hypersurface contained in $M$ through $p_{0}$. Recall the theorem of Trépreau [T] that if $M$ is minimal at $p_{0}$, then every CR function defined on $M$ near $p_{0}$ extends holomorphically to at least one side of $M$ in $\mathbb{C}^{n}$ near $p_{0}$. A stronger condition on a hypersurface $M$ at a point $p_{0}$ is that of essential finiteness (as defined in [BJT], [BR3], [DA2]). We will recall this definition in $\S 1$. We note here that if $M$ is of D'Angelo finite type at $p_{0}$ [DA1], then $M$ is essentially finite at $p_{0}$ (and hence minimal at $p_{0}$ ).

In this paper we prove a general result of the "Hopf lemma" type for CR mappings, with nonidentically vanishing Jacobians, between real hypersurfaces in $\mathbb{C}^{n}$. Applications of this result to finiteness and holomorphic extendibility of such mappings are also given. The novelty here is that we make no assumption on the nonflatness of the mapping or its Jacobian, nor do we assume that the hypersurfaces are pseudoconvex or minimally convex.

Theorem 1. Let $M$ be a smooth, connected, orientable hypersurface in $\mathbb{C}^{n}$ which is essentially finite at all points. Let $h: M \rightarrow M^{\prime}$ be a smooth $C R$ mapping from $M$ to another smooth hypersurface $M^{\prime} \subset \mathbb{C}^{n}$, with Jac $h \not \equiv 0$.

Received April 17, 1995.

The first and third authors were partially supported by National Science Foundation Grant DMS 9203973. 
Let $p_{0} \in M$, and suppose that $h^{-1}\left(h\left(p_{0}\right)\right)$ is a compact subset of $M$. Then $h$ satisfies the Hopf lemma property at $p_{0}$.

If the hypersurfaces are pseudoconvex, the result above follows from the classical Hopf lemma for harmonic functions, as proved in Fornaess $[\mathrm{F}]$ (see also $[\mathrm{BBR}]$ ). Other results of the Hopf lemma type for CR mappings were previously obtained in [BR3] and [BR5].

As shown in [BBR], [BR1], and [DF], the Hopf lemma property can be used to prove holomorphic extension of $\mathrm{CR}$ mappings between real analytic hypersurfaces. From Theorem 1 we obtain the following corollary.

Corollary 1. Let $M, M^{\prime}, h$ be as in Theorem 1, and assume in addition that $M$ and $M^{\prime}$ are real analytic. Then $h$ extends holomorphically to a neighborhood of $p_{0}$ in $\mathbb{C}^{n}$.

In the global case, i.e. when $M$ and $M^{\prime}$ are compact boundaries, the compactness of $h^{-1}\left(h\left(p_{0}\right)\right)$ is automatically satisfied, yielding the following result.

Theorem 2. Let $\Omega$ and $\Omega^{\prime}$ be bounded domains in $\mathbb{C}^{n}$ with smooth boundaries, such that $\partial \Omega$ is essentially finite at all points. Suppose $H: \Omega \rightarrow \Omega^{\prime}$ is a proper holomorphic mapping, smooth up to $\partial \Omega$. Then $H$ satisfies the Hopf lemma property at every point $p \in \partial \Omega$. Furthermore, $H$ is finite-to-one on $\bar{\Omega}$.

When $\Omega$ and $\Omega^{\prime}$ in Theorem 2 are real analytic, we obtain a new proof of the following result of the second author and Pan $[\mathrm{HP}]$, extending earlier results in [BR1], [DF], [BR3].

Corollary 2. If $\Omega$ and $\Omega^{\prime}$ are bounded domains in $\mathbb{C}^{n}$ with real analytic boundaries, and $H: \Omega \rightarrow \Omega^{\prime}$ is a proper holomorphic mapping, smooth up to $\partial \Omega$, then $H$ extends holomorphically to a neighborhood of $\bar{\Omega}$ in $\mathbb{C}^{n}$.

It should be noted that Theorem 2 and Corollary 2 may be proved more directly (see Remark 2.2).

Another application of Theorem 1 is a propagation result of the Hopf lemma property (Theorem 3), as well as real analyticity (Corollary 3), analogous to the classical Hartog's theorem for extension of holomorphic functions.

Theorem 3. Let $M$ be a smooth, orientable, connected hypersurface in $\mathbb{C}^{n}$ which is essentially finite at all points, and let $h: M \rightarrow M^{\prime}$ be a smooth CR mapping from $M$ to another smooth hypersurface $M^{\prime} \subset \mathbb{C}^{n}$, with Jac $h \not \equiv 0$. Suppose that $U_{1}$ and $U$ are relatively compact open subsets of $M$, with $\bar{U}_{1} \subset U$. Then if the Hopf lemma property holds at every point in $U \backslash U_{1}$, it also holds everywhere in $U$. 
Corollary 3. Let $M$ be a real analytic, orientable, connected hypersurface in $\mathbb{C}^{n}$ which is essentially finite at all points, and let $h: M \rightarrow M^{\prime}$ be a smooth $C R$ mapping from $M$ to another real analytic hypersurface $M^{\prime} \subset$ $\mathbb{C}^{n}$, with Jac $h \not \equiv 0$. Suppose that $U_{1}$ and $U$ are relatively compact open subsets of $M$, with $\bar{U}_{1} \subset U$. If $h$ is real analytic in $U \backslash U_{1}$, then $h$ is real analytic everywhere in $U$ and hence extends holomorphically to an open neighborhood of $U$ in $\mathbb{C}^{n}$.

Remark 0.1. In Theorem 1 and Corollary 1 , the condition Jac $h \not \equiv 0$ may be replaced by the stronger condition that $M^{\prime}$ does not contain any nontrivial complex variety through $p_{0}$. (See e.g. [BR4].) A similar statement holds for Theorem 3 and Corollary 3.

Some of the results of the present paper, including Corollary 1, were announced earlier by the second author. Also, a recent preprint of Y. Pan $[\mathrm{P}]$ contains a special case of Corollary 1 above and other related results.

\section{$\S 1$ Preliminaries}

Let $M$ be a smooth real hypersurface in $\mathbb{C}^{n}$. For $p \in M$, we denote by $T_{p} M$ the real tangent space of $M$ at $p$ and by $\mathbb{C} T_{p} M$ its complexification. We denote by $\mathcal{V}_{p} M$ the complex subspace of $\mathbb{C} T_{p} M$ consisting of all antiholomorphic vectors tangent to $M$ at $p$, and by $T_{p}^{c} M=\operatorname{Re} \mathcal{V}_{p} M$ the complex tangent space of $M$ at $p$ considered as a real subspace of $T_{p} M$. Note that if $h$ is a smooth CR map from $M$ to a hypersurface $M^{\prime}$, then $h$ satisfies the Hopf lemma property mentioned in $\S 0$ if and only if

$$
d h\left(T_{p_{0}} M\right) \not \subset T_{h\left(p_{0}\right)}^{c} M^{\prime} .
$$

Note that for this form of the definition, it is not necessary to assume that $h$ extends holomorphically to one side of $M$.

If $\rho(z, \bar{z})$ is a defining function for $M$ near $p_{0}=0$, with $\rho(0)=0$ and $d \rho(0) \neq 0$, we consider the formal Taylor series of $\rho$ in $z$ and $\bar{z}$ at 0 and write $R(z, \zeta)$ for its complexificiation, i.e. $R(z, \zeta) \equiv \sum a_{\alpha, \beta} z^{\alpha} \zeta^{\beta}$, where $\alpha ! \beta ! a_{\alpha \beta}=\rho_{z^{\alpha} \bar{z}^{\beta}}(0)$. Let $X_{1}, \ldots, X_{n}$, be the vector fields with formal power series coefficients given by

$$
X_{j}=R_{\zeta_{n}}(0, \zeta) \frac{\partial}{\partial \zeta_{j}}-R_{\zeta_{j}}(0, \zeta) \frac{\partial}{\partial \zeta_{n}}, \quad j=1, \ldots, n-1
$$

where we have assumed $\rho_{z_{n}}(0) \neq 0$. For a multi-index $\alpha=\left(\alpha_{1}, \ldots, \alpha_{n-1}\right)$ we define $c_{\alpha}(z)$ in the ring of convergent power series in $n$ complex variables, by

$$
c_{\alpha}(z)=\left.X^{\alpha} R(z, \zeta)\right|_{\zeta=0},
$$


where $X^{\alpha}=X_{1}^{\alpha_{1}} \cdots X_{n-1}^{\alpha_{n-1}}$. We say that $M$ is essentially finite at 0 if the ideal $\left(c_{\alpha}(z)\right)$ generated by the $c_{\alpha}(z)$ in the ring $\mathbb{C}[[z]]$ of formal power series is of finite codimension. It should be noted that this definition is independent of the choice of coordinates and defining function $\rho$; it is given in a slightly different form in [BR3]. Note also that if $M$ is essentially finite at $p_{0}$, then $M$ is minimal at $p_{0}$, and that if $M$ is of D'Angelo finite type, then it is essentially finite.

Recall that an analytic disc in $\mathbb{C}^{n}$ is a continuous mapping $A: \bar{\Delta} \rightarrow \mathbb{C}^{n}$ which is holomorphic in $\Delta$, where $\Delta$ is the open unit disc in the plane. We say that $A$ is attached to $M$ if $A(\partial \Delta) \subset M$. Let $M$ be a smooth hypersurface minimal at $p_{0}$. As in [BR5], we say that $M$ is minimally convex at $p_{0}$ if $M$ is minimal at $p_{0}$, and there is a neighborhood $U$ of $p_{0}$ in $M$ and a side of the hyperplane $T_{p_{0}} M$ in $\mathbb{C}^{n}$ such that the real derivatives $\frac{\partial}{\partial \xi}[A(\xi)]_{\left.\right|_{\xi=1}}$ lie on that side or in $T_{p_{0}} M$, for all sufficiently smooth analytic $\operatorname{discs} A$ attached to $U$ with $A(1)=p_{0}$. Here $\zeta=\xi+i \eta$, with $\zeta \in \Delta$.

For the convenience of the reader we begin by stating a number of known results, Theorems A, B, C and D below, which will be important for the proofs of Theorem 1. Theorem A is a consequence of a result of Tumanov $[\mathrm{Tu}]$, as observed in [BR5] .

Theorem A [Tu, BR5 ]. Let $M$ be a smooth, real hypersurface in $\mathbb{C}^{n}$, and assume that $M$ is minimal at $p_{0}$. Then one of the following two conditions holds.

(1) $M$ is minimally convex at $p_{0}$.

(2) Every $C R$ function defined in a neighborhood of $p_{0}$ in $M$ extends holomorphically to a full neighborhood of $p_{0}$ in $\mathbb{C}^{n}$.

Theorem B [BR5]. If $h$ is a smooth CR mapping from a smooth hypersurface $M$ to another smooth hypersurface $M^{\prime}$, with $M$ minimal at $p_{0}$, Jac $h \not \equiv 0$, and $M^{\prime}$ minimally convex at $p_{0}^{\prime}=h\left(p_{0}\right)$, then the Hopf lemma property holds at $p_{0}$.

We need also to recall a result which follows from Theorem 4 in [BR3].

Theorem C [BR3]. Let $H$ be a holomorphic map defined in a neighborhood of a smooth hypersurface $M$ essentially finite at $p_{0}, H(M) \subset M^{\prime}$, with $M^{\prime}$ another smooth hypersurface of $\mathbb{C}^{n}$, and Jac $H \not \equiv 0$. Then $H$ satisfies the Hopf lemma property at $p_{0}$ and $H$ is finite-to-one in a neighborhood of $p_{0}$.

We shall also need a stronger version of this result, and we indicate here its proof. 
Theorem D. Let $H$ be a holomorphic map defined on one side of a smooth hypersurface $M$ essentially finite at $p_{0}$, with $H$ smooth up to $M$. Suppose $H(M) \subset M^{\prime}$, with $M^{\prime}$ another smooth hypersurface of $\mathbb{C}^{n}$. Then Jac $H$ is not flat at $p_{0}$ if and only if $H$ satisfies the Hopf lemma property at $p_{0}$. In addition, if either of these equivalent conditions is satisfied, then any smooth extension of $H$ to a sufficiently small neighborhood of $p_{0}$ in $\mathbb{C}^{n}$ is finite-to-one.

Proof of Theorem D. If Jac $H$ is not flat at $p_{0}$, we conclude that if $G$ is a formal transversal component of $H$ (as defined in [BR3]), then $G \not \equiv 0$. Hence, by Theorem 4 of [BR3], it follows that $H$ satisfies the Hopf lemma property and is of finite multiplicity. Conversely, if $H$ satisfies the Hopf Lemma property at $p_{0}$, by Theorem 4 of [BR3] it follows again that $H$ is of finite multiplicity and also that $M^{\prime}$ is essentially finite at $H\left(p_{0}\right)$. By Theorem 3 of [BR3], we conclude also that $H$ is not totally degenerate at $p_{0}$, in the sense of [BR3], and hence, using again the Hopf lemma property, Jac $H$ is not flat at $p_{0}$.

We may assume $p_{0}=H\left(p_{0}\right)=0$. Since $H$ is holomorphic on one side of $M$ and smooth up to the boundary, its Taylor series at 0 defines a formal (not necessarily convergent) holomorphic map $H=\left(\sum a_{\alpha}^{1} z^{\alpha}, \ldots, \sum a_{\alpha}^{n} z^{\alpha}\right)$. The equivalent conditions above imply that $H$ is finite as a formal map. That is, the ideal generated by the $\sum a_{\alpha}^{j} z^{\alpha}, j=1, \ldots, n$, is of finite codimension in the ring $\mathbb{C}[[z]]$ of formal power series in $z$. Since the Taylor series of $H$ coincides with that of any smooth extension of $H$ to $\mathbb{C}^{n}=\mathbb{R}^{2 n}$, we conclude e.g. by [GG], [EL], that this extension is finite-to-one near 0 .

\section{$\S 2$ Inverse image of a nonminimally convex point.}

In this section we shall state and prove a new result, Theorem 4 below, from which Theorem 1 will follow.

Theorem 4. Let $h: M \rightarrow M^{\prime}$ be a CR map, where $M, M^{\prime}, h, p_{0} \in$ $M$ satisfy all the conditions of Theorem 1. In addition, suppose that $M^{\prime}$ is not minimally convex at $p_{0}^{\prime}=h\left(p_{0}\right)$. Then all CR functions on $M$ extend holomorphically to a full neighborhood of $p_{0}$ in $\mathbb{C}^{n}$. In particular, $h$ extends holomorphically to a neighborhood of $p_{0}$ and satisfies the Hopf lemma property at $p_{0}$.

Before proving Theorem 4, we note that Theorem 1 is a consequence of Theorem 4 and Theorem B above. Indeed, if $M^{\prime}$ is minimally convex at $h\left(p_{0}\right)$, then since any essentially finite hypersurface is minimal at all points, Theorem 1 follows from Theorem B. On the other hand, if $h\left(p_{0}\right)$ is 
not minimally convex, Theorem 1 is an immediate consequence of Theorem 4 .

In the rest of this section, we shall prove Theorem 4 . We may assume that $p_{0}=p_{0}^{\prime}=0$, and we let $Z_{M}=h^{-1}(0)$. Note that $Z_{M}$ is a compact subset of $M$ by the assumptions of the theorem. Hence without loss of generality, we shall assume that $M$ is bounded.

The following lemma shows that we can reduce the proof of the theorem to the case where $h$ extends holomorphically to one side of $M$.

Lemma 2.1. Under the assumptions of Theorem 1, there exists an open neighborhood $\mathcal{U}$ of 0 in $\mathbb{C}^{n}$ such that $Z_{M} \cap \mathcal{U}$ is compact in $M \cap \mathcal{U}$ and $h$ extends holomorphically to $\mathcal{U}^{+}$, one side of $M$ in $\mathcal{U}$.

Proof. Since $M$ is essentially finite and hence minimal at all points, it follows that $h$ extends holomorphically to at least one side of $M$ at each point. Since $M$ is orientable, it is given by a global smooth defining function $\rho$ with nonvanishing gradient on $M$. We may assume that $h$ extends to the plus side of $M$, ( i.e. where $\rho(z)>0$ ) near 0 . Let $M_{1}$ be the largest connected open subset of $M$ containing 0 such that $h$ extends holomorphically to the plus side of $M$ near every point of $M_{1}$.

If $M_{1}=M$, then the Lemma is an immediate consequence of the assumptions of Theorem 1. Assume therefore that $M_{1}$ is a proper subset of $M$ and let $\partial M_{1}$ be its boundary in $M$. For $\delta>0$, let $M_{1}^{\delta}=\left\{p \in M_{1}\right.$ : $\left.\operatorname{dist}\left(p, \partial M_{1}\right)>\delta\right\}$. Since at every point of $M, h$ extends holomorphically to at least one side of $M$, it follows from the definition of $M_{1}$ that there is an open neighborhood $U$ of $\partial M_{1}$ in $M$ such that $h$ extends holomorphically to both sides of $M$ at every point in $U \cap M_{1}$. Applying Theorem $\mathrm{C}$, we conclude that $Z_{M} \cap U \cap M_{1}$ is a discrete set.

Let $\partial M$ be the boundary of $M$ in $\mathbb{C}^{n}$ and choose $a>0$ such that $a<\operatorname{dist}\left(Z_{M}, \partial M\right)$ (which is possible by the assumption of the theorem). Denote by $M^{a}=\{p \in M: \operatorname{dist}(p, \partial M)>a\}$. Note that $\partial M_{1} \cap \overline{M^{a}}$ is compact in $M$. Therefore, there exists $\delta_{0}>0$ such that for all $\delta, 0<\delta<\delta_{0}$, we have

$$
\partial M_{1}^{\delta} \cap Z_{M}=\overline{M^{a}} \cap \partial M_{1}^{\delta} \cap Z_{M} \subset U \cap M_{1} .
$$

By compactness and the discreteness mentioned above, we conclude that $\partial M_{1}^{\delta} \cap Z_{M}$ is a finite set. Since these sets are all disjoint for different $\delta$ ' $s$, we conclude that there exists $\delta_{1}$, with $0<\delta_{1}<\delta_{0}$, such that $\partial M_{1}^{\delta_{1}} \cap Z_{M}=$ $\emptyset$. Now the lemma follows by taking $\mathcal{U}$ to be a sufficiently small open neighborhood of $M_{1}^{\delta_{1}}$ in $\mathbb{C}^{n}$.

By Lemma 2.1, after shrinking $M$ if necessary, we may now assume that there is a connected open set $\mathcal{O}$ in $\mathbb{C}^{n}$ such that:

(i) $\mathcal{O} \cup M$ is a manifold with boundary of class $C^{\infty}$. 
(ii) $h$ extends holomorphically to $\mathcal{O}$; if $H$ denotes the holomorphic extension of $h$, then $H \in C^{\infty}(\overline{\mathcal{O}})$.

(iii) $h(0)=0$.

(iv) $Z_{M}=h^{-1}(0)$ is a compact subset of $M$.

We write

$$
Z=H^{-1}(0) \cap \mathcal{O} .
$$

We shall show that we can take $H$ to be a proper mapping of an open domain to its image. The following lemma is crucial in this construction.

Lemma 2.2. Let $V$ be a connected open neighborhood of $Z_{M}$ in $M$, with $\bar{V}$ a compact subset of $M$. For $\delta>0$, let

$$
\mathcal{O}^{\delta}=\{z \in \mathcal{O}: \operatorname{dist}(z, \bar{V})<\delta\}
$$

and $\partial \mathcal{O}^{\delta}=S_{1}^{\delta} \cup S_{2}^{\delta}$, with $S_{1}^{\delta}=\partial \mathcal{O}^{\delta} \cap M$ and $S_{2}^{\delta}=\partial \mathcal{O}^{\delta} \backslash S_{1}^{\delta}$. Then for any $\delta_{0}>0$ there exists $\delta, 0<\delta<\delta_{0}$, such that $S_{2}^{\delta} \cap Z=\emptyset$.

Proof. Note that by assumption, $\overline{S_{2}^{\delta}} \cap Z_{M}=\emptyset$. Hence there exists $\epsilon>0$ such that for any $\delta$ sufficiently small,

$$
Z \cap\left\{z \in S_{2}^{\delta}: \operatorname{dist}\left(z, \overline{S_{2}^{\delta}} \cap M\right)<\epsilon\right\}=\emptyset .
$$

Let $Z^{\prime}=\{z \in Z: z$ is not an isolated point of $Z\}$. If there exists $\delta>0$ such that $Z^{\prime} \cap \mathcal{O}^{\delta}=\emptyset$, then $Z \cap \mathcal{O}^{\delta}$ is countable, and the conclusion of the lemma follows since the sets $S_{2}^{\delta}$, as $\delta$ varies, are disjoint.

To complete the proof of the lemma, we shall assume

$$
Z^{\prime} \cap \mathcal{O}^{\delta} \neq \emptyset
$$

for all $\delta$ sufficiently small, and reach a contradiction. It is clear that under condition $(2.5)$ we have

$$
\overline{Z^{\prime}} \cap M \neq \emptyset .
$$

We claim that Jac $H$ vanishes to infinite order at every point $p \in \overline{Z^{\prime}} \cap M$. Indeed if Jac $H$ does not vanish to infinite order at a point $p \in Z_{M}$, then by Theorem D, $p$ is an isolated point of $H^{-1}(0)$ in $\overline{\mathcal{O}}$. Since this cannot be the case for $p \in \overline{Z^{\prime}} \cap M \subset Z_{M}$, the claim follows.

Now let $T_{2}^{\delta}=\left\{z \in S_{2}^{\delta}: \operatorname{dist}\left(z, \overline{S_{2}^{\delta}} \cap M\right) \geq \epsilon\right\}$, where $\epsilon$ satisfies (2.4). Using (2.4), we note that

$$
\overline{Z^{\prime}} \cap \partial \mathcal{O}^{\delta} \subset T_{2}^{\delta} \cup\left(Z_{M} \cap \overline{Z^{\prime}}\right) .
$$


Since $T_{2}^{\delta}$ is compactly contained in $\mathcal{O}$ for sufficiently small $\delta$, and Jac $H$ is holomorphic in $\mathcal{O}$, there exists $C>0$ such that for all multi-indices $\alpha$

$$
\sup _{z \in T_{2}^{\delta}}\left|D^{\alpha} \operatorname{Jac} H(z)\right| \leq C^{|\alpha|+1} \alpha !
$$

By the maximum principle on complex varieties (see e.g. [N1]) we have,

$$
\sup _{z \in \overline{Z^{\prime}} \cap \overline{\mathcal{O}^{\delta}}}\left|D^{\alpha} \operatorname{Jac} H(z)\right|=\sup _{z \in \overline{Z^{\prime}} \cap \partial \mathcal{O}^{\delta}}\left|D^{\alpha} \operatorname{Jac} H(z)\right|
$$

However, as proved in the claim above, Jac $H$ vanishes to infinite order on $\overline{Z^{\prime}} \cap M$. Hence, in view of (2.7) and (2.8)

$$
\sup _{z \in \overline{Z^{\prime}} \cap \overline{\mathcal{O}^{\delta}}}\left|D^{\alpha} \operatorname{Jac} H(z)\right|=\sup _{z \in T_{2}^{\delta}}\left|D^{\alpha} \operatorname{Jac} H(z)\right| \leq C^{|\alpha|+1} \alpha ! .
$$

This proves that the radius of convergence of Jac $H(z), z \in Z^{\prime}$, is greater than a positive constant which is independent of the distance to $M$. Hence Jac $H$ extends holomorphically to a full neighborhood in $\mathbb{C}^{n}$ of each point of $\overline{Z^{\prime}} \cap M$. Since Jac $H$ vanishes to infinite order there, it follows that Jac $H \equiv 0$, contrary to assumption. We conclude that (2.6), and hence (2.5), cannot hold, which completes the proof of Lemma 2.2.

In reducing the proof of Theorem 4 to the global case of a proper mapping we shall use the following.

Proposition 2.10. Let $M$ be a connected hypersurface of class $C^{0}$ with $M \subset \partial \mathcal{O}$, where $\mathcal{O}$ is an open bounded domain in $\mathbb{C}^{n}$, and let $H$ be a holomorphic mapping in $\mathcal{O}$, continuous up to the boundary, Jac $H \not \equiv 0$, with $H(M)$ contained in another hypersurface $M^{\prime}$ of class $C^{0}$. Suppose $0 \in M, H(0)=0$, and

$$
H^{-1}(0) \cap \partial \mathcal{O} \subset M
$$

Then there is a subdomain $\mathcal{O}_{1} \subset \mathcal{O}$ satisfying

(i) $0 \in \partial \mathcal{O}_{1}$, and there exists a sequence $\left\{z_{j}\right\} \subset \mathcal{O}_{1}$ such that $z_{j} \rightarrow 0$ and $H\left(z_{j}\right)$ stays strictly on one side of $M^{\prime}$;

(ii) there exists $U$, a neighborhood of 0 in $M^{\prime}$, with $\overline{H\left(\mathcal{O}_{1}\right)} \supset U$;

(iii) $H: \mathcal{O}_{1} \rightarrow H\left(\mathcal{O}_{1}\right)$ is a proper map.

Proof. We begin with the following lemma, which describes a well-known construction, see e.g. [BC]. 
Lemma 2.12. Let $\mathcal{O} \subset \mathbb{C}^{n}$ be an open bounded domain, and suppose $H: \mathcal{O} \rightarrow \mathbb{C}^{n}$ is a holomorphic mapping, continuous up to $\partial \mathcal{O}$. Let

$$
D=\{z \in \mathcal{O}: H(z) \notin H(\partial \mathcal{O})\}
$$

If $D \neq \emptyset$, then $H: D \rightarrow H(D)$ is finite-to-one and hence open. Furthermore, if $D_{1}^{\prime}$ is any connected component of $H(D)$, and $D_{1}$ a connected component of $H^{-1}\left(D_{1}^{\prime}\right)$, then $H: D_{1} \rightarrow D_{1}^{\prime}$ is a proper map.

Proof. Since this result is in the "folklore", we shall be brief. We assume $D \neq \emptyset$. If $H$ is not finite-to-one, there exists $w \in H(D)$ for which $H^{-1}(w)$ has an accumulation point $z_{0}$ in $\bar{D}$ (and hence $H\left(z_{0}\right)=w$ ). By the definition of $D$,

$$
H(D) \cap H(\partial \mathcal{O})=\emptyset, \text { and } H(\partial D) \subset H(\partial \mathcal{O})
$$

Hence $z_{0} \notin \partial D$. On the other hand, if $z_{0} \in D$, then there is a nontrivial variety contained in $H^{-1}(w)$, which would necessarily intersect $\partial D$. Since this is also impossible, by the definition of $D, H$ is finite-to-one and hence open (see e.g. $[\mathrm{R}]$ ).

To show that $H: D_{1} \rightarrow D_{1}^{\prime}$ is proper, suppose $z_{j} \rightarrow z_{0}, z_{j} \in D_{1}$, $z_{0} \in \partial D_{1}$. Then by continuity $H\left(z_{j}\right) \rightarrow H\left(z_{0}\right)=w_{0} \in \overline{D_{1}^{\prime}}$. We claim that $w_{0} \in \partial D_{1}^{\prime}$. Indeed, if $w_{0}$ is an interior point of $D_{1}^{\prime}$, let $V^{\prime}$ be an neighborhood of $w_{0}$ in $D_{1}^{\prime}$. Consider $H$ as a map from $\overline{\mathcal{O}}$ to $\mathbb{C}^{n}$. Then a component of $H^{-1}\left(V^{\prime}\right)$ is contained in $D_{1}$, by the definition of $D$. Then $z_{0}$ would be an interior point of $D_{1}$, contrary to assumption. This proves Lemma 2.12 .

We may now complete the proof of Proposition 2.10. Let $\rho^{\prime}$ be a defining function for $M^{\prime}$ near 0 . Without loss of generality, we may assume that there exist $z_{j} \in \mathcal{O}, j=1,2, \ldots$, with

$$
\lim _{j} z_{j}=0 \text { and } \rho^{\prime}\left(H\left(z_{j}\right)\right)>0 .
$$

Indeed, we first select $z_{j} \in \mathcal{O}$ with Jac $H\left(z_{j}\right) \neq 0$. Since $H$ is open near such a $z_{j}$, by slightly moving $z_{j}$ if necessary, we may assume $H\left(z_{j}\right) \notin M^{\prime}$. Replacing $\rho^{\prime}$ by $-\rho^{\prime}$ and selecting a subsequence if necessary, we reach the desired conclusion (2.14).

Let $D$ be as in Lemma 2.12. By hypothesis (2.11) and the continuity of $H$ it follows that $H(\partial \mathcal{O} \backslash M)$ is a compact set which does not contain 0 . Hence, by taking $z_{j}$ sufficiently close to 0 , we may assume that the points $z_{j}$ 
chosen in (2.14) are in $D$. We shall show that there exists $\epsilon>0$, arbitrarily small, such that

$$
\left\{w \in \mathbb{C}^{n}:|w|<\epsilon \text { and } \rho^{\prime}(w)>0\right\} \equiv W_{\epsilon} \subset H(D)
$$

Suppose that (2.15) is proved. Let $D_{1}^{\prime}$ be the connected component of $H(D)$ containing the connected open set $W_{\epsilon}$. We claim that there is a connected component $D_{1}$ of $H^{-1}\left(D_{1}^{\prime}\right)$ such that $0 \in \partial D_{1}$. Indeed, by Lemma 2.12, the restriction of $H$ to $D$ is finite-to-one, and the restriction to any connected component of $H^{-1}\left(D_{1}^{\prime}\right)$ is proper, and hence onto $D_{1}^{\prime}$. Therefore, $H^{-1}\left(D_{1}^{\prime}\right)$ consists of finitely many connected components $D_{k}$. Choose one of these components, say $D_{1}$, which contains infinitely many of the $z_{j}$. Then $0 \in \partial D_{1}$. Since, by Lemma 2.12, the restriction of $H$ to $D_{1}$ is proper onto $H\left(D_{1}\right)=D_{1}^{\prime}$, Proposition 2.10 will follow by taking $\mathcal{O}_{1}=D_{1}$.

It remains to prove (2.15). Choose $\epsilon$ such that

$$
0<\epsilon<\operatorname{dist}(0, H(\partial \mathcal{O} \backslash M))
$$

and such that the open set $W_{\epsilon}$ defined in (2.15) is connected. Let $j_{0}$ be such that $H\left(z_{j_{0}}\right) \in W_{\epsilon}$. Let $w \in W_{\epsilon}$ be arbitrary, and $\gamma(t), 0 \leq t \leq 1$, be a continuous curve connecting $H\left(z_{j_{0}}\right)$ and $w$ and contained in $W_{\epsilon}$. Assume by contradiction that $w \notin H(D)$. Since $H(D)$ is open, there exists $t^{\prime}, 0<t^{\prime} \leq$ 1 , such that $\gamma(t) \in H(D)$ for $0 \leq t<t^{\prime}$, but $\gamma\left(t^{\prime}\right) \notin H(D)$. Now choose a sequence $t_{k}<t^{\prime}$, with $t_{k} \rightarrow t^{\prime}$, and $p_{k} \in D$ with $H\left(p_{k}\right)=\gamma\left(t_{k}\right)$ and $p_{k} \rightarrow p^{\prime} \in \bar{D}$. Since $H\left(p^{\prime}\right)=\gamma\left(t^{\prime}\right) \notin H(D)$, it follows from the definition of $D$ that $p^{\prime} \in \partial D$. Recall that $H(\partial D) \subset H(\partial \mathcal{O})$. Hence $H\left(p^{\prime}\right) \in H(\partial \mathcal{O})$. In view of (2.16) and the fact that $H$ maps $M$ into $M^{\prime}$, we must have $H\left(p^{\prime}\right) \in M^{\prime}$. We reach a contradiction, since $H\left(p^{\prime}\right)=\gamma\left(t^{\prime}\right) \in W_{\epsilon}$. The proof of Proposition 2.10 is now complete.

Let $\Omega$ be a bounded domain in $\mathbb{C}^{n}$ and $p_{0} \in \mathbb{C}^{n}$. Recall that $p_{0}$ is in the holomorphic hull of $\Omega$ if there is a compact subset $K \subset \Omega$ such that $p_{0} \in \overline{\hat{K}}$, where

$$
\hat{K}=\left\{z \in \Omega:|f(z)| \leq \sup _{w \in K}|f(w)| \text { for all } f \in \mathcal{H}(\Omega)\right\}
$$

Here $\mathcal{H}(\Omega)$ denotes the space of all holomorphic functions in $\Omega$. We observe that when $p_{0}$ is a boundary point of $\Omega$, then $p_{0}$ is in the holomorphic hull of $\Omega$ if and only if any function in $\mathcal{H}(\Omega)$ extends holomorphically to some larger domain which contains $p_{0}$ as an interior point. 
Proposition 2.17. Let $\Omega$ and $\Omega^{\prime}$ be two bounded domains in $\mathbb{C}^{n}$ and $H$ a proper holomorphic mapping from $\Omega$ to $\Omega^{\prime}$. Suppose that $p_{0}$ and $p_{0}^{\prime}$ are boundary points of $\Omega$ and $\Omega^{\prime}$ respectively, and that there is a sequence $\left\{z_{j}\right\}_{j=1}^{\infty} \subset \Omega$ converging to $p_{0}$ such that $\lim _{j} H\left(z_{j}\right)=p_{0}^{\prime}$. Suppose that any function in $\mathcal{H}\left(\Omega^{\prime}\right)$ is bounded on the sequence $\left\{H\left(z_{j}\right)\right\}_{j=1}^{\infty}$. Then $p_{0}$ is in the holomorphic hull of $\Omega$.

Remark 2.18. Note that the hypothesis of the proposition is satisfied if $p_{0}^{\prime}$ is in the envelope of holomorphy of $\Omega^{\prime}$.

Proof. By using a standard result (see e.g. [N2] Chapter 7, Lemma 2), it suffices to prove the following claim:

Each function in $\mathcal{H}(\Omega)$ is bounded on $\left\{z_{j}\right\}$. More precisely, for any $f \in \mathcal{H}(\Omega)$, there exists a constant $C_{f}>0$ such that $\left|f\left(z_{j}\right)\right| \leq C_{f}$ for all $j$.

To prove the claim, we note that $H$ is finite-to-one on $\Omega$ since it is proper. Hence there exists $m$ such that each $w \in \Omega^{\prime}$ has $m$ pre-images, $g_{k}(w), k=1 \ldots m$, counted with multiplicity (see e.g. $[\mathrm{R}]$ ). Now let $f \in$ $\mathcal{H}(\Omega)$ and denote by $\sigma_{1}(w), \ldots, \sigma_{m}(w)$ the elementary symmetric functions of $f\left(g_{k}(w)\right), k=1, \ldots, m$. By well known results (see e.g. $[\mathrm{R}]$ ) the $\sigma_{k}(w)$ are holomorphic in $\Omega^{\prime}$ and hence, by hypothesis, uniformly bounded on the sequence $\left\{H\left(z_{j}\right)\right\}$. If we let $w_{j}=H\left(z_{j}\right)$, we observe that $f\left(z_{j}\right)$ is one of the roots of the polynomial $X^{m}-\sigma_{1}\left(w_{j}\right) X^{m-1}+\ldots+(-1)^{m} \sigma_{m}\left(w_{j}\right)$. Since the coefficients of this polynomial are bounded, independently of $j$, it follows that the $f\left(z_{j}\right)$ are bounded, independently of $j$. This proves the claim and hence Proposition 2.17.

Proof of Theorem 4. First, we prove that under the assumptions of Theorem $4, M^{\prime}$ is minimal at $p_{0}^{\prime}=h\left(p_{0}\right)$. Indeed, suppose not. Then there is a complex hypersurface $\Sigma$ contained in $M^{\prime}$ through $p_{0}^{\prime}$. Hence, this hypersurface must contain all small analytic disks $A^{\prime}$ attached to $M^{\prime}$ with $A^{\prime}(1)=p_{0}^{\prime}$. On the other hand since $M$ is minimal at $p_{0}$, the boundaries of small analytic discs $A$ attached to $M$ with $A(1)=p_{0}$ cover a full neighborhood of $p_{0}$ in $M[\mathrm{Tu}]$. Since we can take $A^{\prime}=h \circ A$, this contradicts the assumption that Jac $h \not \equiv 0$. (See also $[\mathrm{E}]$ for related results.)

By Lemma 2.1, we may assume that $h$ admits a holomorphic extension $H$ to one side of $M$, and that conditions (i)-(iv) preceding Lemma 2.2 are satisfied, so that we may apply Lemma 2.2. If $\delta$ satisfies the conclusion of Lemma 2.2, then $H$ satisfies the hypotheses of Proposition 2.10 with $\mathcal{O}=\mathcal{O}^{\delta}$. We then obtain from Proposition 2.10 a subdomain $\mathcal{O}_{1}$ of $\mathcal{O}$ such that the restriction of $H$ to $\mathcal{O}_{1}$ is a proper mapping from $\mathcal{O}_{1}$ to $\mathcal{O}_{1}^{\prime}$, continuous up to the boundary, with $p_{0} \in \partial \mathcal{O}_{1}$ and $p_{0}^{\prime}=H\left(p_{0}\right) \in \partial \mathcal{O}_{1}^{\prime}$. Moreover, there exists a sequence $\left\{z_{j}\right\} \subset \mathcal{O}_{1}$ such that $w_{j}=H\left(z_{j}\right) \rightarrow p_{0}^{\prime}$, with $\left\{w_{j}\right\}$ strictly on one side of $M^{\prime}$. 
Since $M^{\prime}$ is minimal, but not minimally convex at $p_{0}^{\prime}$, by assumption, it follows from Theorem A that any CR function defined near $p_{0}^{\prime} \in M^{\prime}$ extends holomorphically to a full neighborhood of $p_{0}^{\prime}$ in $\mathbb{C}^{n}$. Now, by using the Baire Category Theorem (see e.g. [BR2, Theorems 7 and 8] for a more general result) we conclude that there is a connected neighborhood $\mathcal{U}^{\prime}$ of $p_{0}^{\prime}$ in $\mathbb{C}^{n}$ with $\mathcal{U}^{\prime} \cap \mathcal{O}_{1}^{\prime} \neq \emptyset$ such that every function in $\mathcal{H}\left(\mathcal{O}_{1}^{\prime}\right)$ extends holomorphically to $\mathcal{U}^{\prime}$. In particular, we see that any such function is uniformly bounded on $\left\{w_{j}\right\}$. Using Proposition 2.17 we conclude that $p_{0}$ is in the holomorphic hull of $\mathcal{O}_{1}$, which lies on the side of $M$ to which every CR function near $p_{0}$ extends. It follows immediately that every CR function near $p_{0}$ on $M$ extends holomorphically to a full neighborhood of $p_{0}$ in $\mathbb{C}^{n}$. The Hopf lemma property then follows from Theorem $\mathrm{C}$ above. The proof of Theorem 4 (and hence that of Theorem 1) is now complete.

\section{$\S 3$ Consequences of Theorem 1 and remarks}

In this section we prove the other results stated in the introduction and make some remarks.

We first note that Corollary 1 follows easily from Theorem 1 and the following holomorphic extendibility result, which is a consequence of Theorem 1 of $[\mathrm{BR} 1]$ :

Theorem E [BR1]. Let $h: M \rightarrow M^{\prime}$ be a smooth $C R$ map, with $M$ and $M^{\prime}$ real analytic hypersurfaces in $\mathbb{C}^{n}$. Assume that $M$ is essentially finite at $p_{0}$ and that $h$ satisfies the Hopf Lemma property at $p_{0}$. Then $h$ extends holomorphically to a full neighborhood of $p_{0}$ in $\mathbb{C}^{n}$.

Proof of Theorem 2. In order to apply Theorem 1, we note first that since $H$ is proper, Jac $H \not \equiv 0$ in $\Omega$. Hence its boundary value on $\partial \Omega$ does not vanish identically. Note also that for any $p_{0} \in \partial \Omega, H^{-1}\left(H\left(p_{0}\right)\right)$ is closed in $\partial \Omega$ and hence compact. We may now conclude by Theorem 1 that the Hopf lemma property holds at each point in $\partial \Omega$.

To prove that $H$ is finite-to-one in $\bar{\Omega}$, we observe first that $H$ is finite-toone in $\Omega$, since it is proper (see e.g. $[R]$ ). Since the Hopf lemma property holds at $p_{0}$, we may apply the last part of Theorem $\mathrm{D}$ to conclude that for any $p_{0} \in \partial \Omega, H$ is finite-to-one in a neighborhood of $p_{0}$ in $\bar{\Omega}$. The desired result then follows by compactness of $\bar{\Omega}$.

Remark 2.1. It also follows from Theorem 4 in [BR3] that under the hypotheses of Theorem $2, \partial \Omega^{\prime}$ is also essentially finite at all points.

Proof of Corollary 2. By a result of Diederich and Fornaess [DF], any compact real analytic boundary in $\mathbb{C}^{n}$ does not contain a nontrivial complex 
variety and hence is essentially finite. We may then apply Theorem 2 to conclude that the Hopf lemma property is satisfied at every point of $M$. The conclusion of Corollary 2 then follows from Theorem E.

Remark 2.2. In fact, Corollary 2 may be proved much more directly by using Proposition 2.17 together with Theorems A, B, C, and E.

Proof of Theorem 3. It suffices to show that if $p_{0} \in U_{1}$, then $h$ satisfies the Hopf lemma property at $p_{0}$. By taking the connected components of $U$ and $U_{1}$ containing $p_{0}$, we may assume, without loss of generality, that $U_{1}$ and $U$ are connected. Let $E=\left\{p \in U: h(p)=h\left(p_{0}\right)\right\}$. Since $M$ is essentially finite, $h$ extends holomorphically to one side of $M$ near any point. Therefore, since by assumption the Hopf lemma property holds in $U \backslash U_{1}$, it follows from Theorem $\mathrm{D}$ that $E \cap\left(U \backslash U_{1}\right)$ is a discrete set.

For $\delta>0$, sufficiently small, let

$$
U^{\delta}=\{p \in U: \operatorname{dist}(p, \partial U)>\delta\},
$$

and let $\partial U^{\delta}$ be its boundary. By the discreteness established above and the compactness of $\partial U^{\delta}$, we conclude that for sufficiently small $\delta$, that $\partial U^{\delta} \cap E$ is finite. Hence there exists $\delta_{1}>0$ for which the set $\partial U^{\delta_{1}} \cap E$ is empty. It is now easy to check that the hypotheses of Theorem 1 are satisfied for $h$ and $p_{0}$ by taking $M=U^{\delta_{1}}$. This proves Theorem 3 .

Proof of Corollary 3. Since $h$ is real analytic at all points of $U \backslash U_{1}$ and $M$ is real analytic, $h$ extends holomorphically to a full neighborhood in $\mathbb{C}^{n}$ of each such point. By Theorem $\mathrm{C}, h$ then satisfies the Hopf lemma property in all of $U \backslash U_{1}$ and hence in all of $U$ by Theorem 3. Applying Corollary 1, we then have that $h$ extends holomorphically to a full neighborhood of $U$ in $\mathbb{C}^{n}$.

\section{References}

[BC] S. Bell and D. Catlin, Regularity of CR mappings, Math. Z. (1988), 357-368.

[BBR] M.S. Baouendi, S. Bell, and L. P. Rothschild, Mappings of three dimensional CR manifolds and their holomorphic extension, Duke Math. J. 56 (1988), 503-530.

[BJT] M. S. Baouendi, H. Jacobwitz, and F. Treves, On the real analyticity of CR mappings, Ann. of Math. 122 (1985), 365-400.

[BR1] M.S. Baouendi and L.P. Rothschild, Germs of CR maps between real analytic hypersurfaces, Invent. Math. 93 (1988), 481-500.

[BR2] M.S. Baouendi and L.P. Rothschild, Extension of holomorphic functions in generic wedges and their wave front sets, Comm. P.D.E. 13 (1988), 1441-1466.

[BR3] , Geometric properties of smooth and holomorphic mappings between surfaces in complex spaces, J. of Diff. Geom. 31 (1990), 473-499.

[BR4] , Remarks on the generic rank of a CR mappings, J. of Geom. Analysis 2 (1992), 1-9. 
[BR5] _ A generalized complex Hopf lemma and its applications to CR mappings, Invent. Math. 111 (1993), 331-348.

[DA1] J. D'Angelo, Real hypersurfaces, orders of contact, and applications, Ann. of Math. 115 (1982), 615-637.

[DA2] J. D'Angelo, The notion of formal essential finiteness for smooth real hypersurfaces, Indiana J. Math. 36 (1987), 897-903.

[DF] K. Diederich, J. Fornaess, Proper holomorphic mappings between real analytic pseudoconvex domains in $\mathbb{C}^{n}$, Math. Ann 282 (1988), 681-700.

[E] P. Ebenfelt, On the unique continuation problem for CR mappings into nonminimal hypersurfaces, to appear, J. of Geom. Analysis.

[EL] D. Eisenbud and H. Levine, An algebraic formula for the degree of a $C^{\infty}$ map germ, Ann. of Math. 106 (1977), 19-44.

[F] J. Fornaess, Biholomorphic mappings between weakly pseudoconvex domains, Pacific J. Math 74 (1978), 63-65.

[GG] M. Golubitsky and V. Guillemin, Stable mappings and their singularities, Springer-Verlag, 1974.

[HP] X. Huang and Y. Pan, Proper holomorphic mappings between real analytic domains in $\mathbb{C}^{n}$, to appear, Duke Math. J.

[N1] R. Narashimhan, Introduction to the Theory of Analytic Spaces, Lecture Notes in Math., Springer 25 (1966).

[N2] R. Narashimhan, Several Complex Variables, University of Chicago Press, 1971.

[P] Y. Pan, A characterization of the finite multiplicity of a CR mapping, preprint.

[R] W. Rudin, Function theory on the unit ball of $\mathbb{C}^{n}$, Springer-Verlag, 1980.

[T] J.-M. Trépreau, Sur le prolongement holomorphe des fonctions CR définies sur une hypersurface réelle de classe $C^{2}$ dans $\mathbb{C}^{n}$, Invent. Math. 83 (1986), 583-592.

[Tu] A. E. Tumanov, Extending CR functions on manifolds of finite type to a wedge, Mat. Sbornik 136 (1988), 128-139.

Department of Mathematics-0112, University of California, La Jolla, Ca 92093-0112

E-mail address: sbaouendi@ucsd.edu

Department of Mathematics, Univerity of Chichao, Chichgo, IL 60637

E-mail address: xhuang@math.uchicago.edu

Department of M athematics-0112, Univerity of California, La Jolla, CA 92093-0112

E-mail address: lrothschild@ucsd.edu 